\title{
Development and Implementation of a Clinical and Business Intelligence System for the Florida Health Data Warehouse
}

\author{
Raed H. AlHazme ${ }^{1}$, Arif M. Rana ${ }^{1}$, Michael De Lucca ${ }^{2}$ \\ 1. Nova Southeastern University College of Osteopathic Medicine, Fort Lauderdale, Florida ${ }^{1}$ \\ 2. Broward Regional Health Planning Council, Hollywood, Florida ${ }^{2}$
}

\begin{abstract}
Objective: To develop and implement a Clinical and Business Intelligence (CBI) system for the Florida Health Data Warehouse (FHDW) in order to bridge the gap between Florida's healthcare stakeholders and the health data archived in FHWD.

Materials and Methods: A gap analysis study has been conducted to evaluate the technological divide between the relevant users and FHWD health data, which is maintained by the Broward Regional Health Planning Council (BRHPC). The study revealed a gap between the health care data and the decision makers that utilize the FHDW data. To bridge the gap, a CBI system was proposed, developed and implemented by BRHPC as a viable solution to address this issue, using the System Development Life Cycle methodology.

Results: The $\mathrm{CBI}$ system was successfully implemented and yielded a number of positive outcomes. In addition to significantly shortening the time required to analyze the health data for decision-making processes, the solution also provided end-users with the ability to automatically track public health parameters.

Discussion: A large amount of data is collected and stored by various health care organizations at the local, state, and national levels. If utilized properly, such data can go a long way in optimizing health care services. $\mathrm{CBI}$ systems provide health care organizations with valuable insights for improving patient care, tracking trends for medical research, and for controlling costs.

Conclusion: The $\mathrm{CBI}$ system has been found quite effective in bridging the gap between Florida's healthcare stake holders and FHDW health data. Consequently, the solution has improved in the planning and coordination of health care services for the state of Florida.

Keywords: Business Intelligence; Clinical Analytics; Data Warehouse; Health Care Planning; Public Health Informatics.

Correspondence: ra556@nova.edu

DOI: $10.5210 /$ ojphi.v6i2.5249

Copyright @2014 the author(s)

This is an Open Access article. Authors own copyright of their articles appearing in the Online Journal of Public Health Informatics. Readers may copy articles without permission of the copyright owner(s), as long as the author and OJPHI are acknowledged in the copy and the copy is used for educational, not-for-profit purposes.
\end{abstract}

\section{Background and Significance}

The project was conducted at the custodian of the Florida Health Data Warehouse (FHDW), Broward Regional Health Planning Council (BRHPC), incorporated, based in Hollywood, 
Florida. BRHPC is a non-profit organization that was established in 1983 under Florida Statute (408.033), as the legislatively designated Broward County local health planning entity. BRHPC provides health and human services at the national, state, and local level through planning, direct services, implementation, evaluation, and organizational capacity building. During the last several years, BRHPC has led statewide collaborative planning activities in partnership with ten other Florida local health-planning councils.

BRHPC has established several databases under the umbrella of their FHDW that provide community members with access to vital health planning and policy making data. Such databases include the Hospital Utilization, Nursing Home Utilization, Florida Prevention Quality Indicators (PQI), and Diagnosis Related Group (DRG) Data Warehouse. The Medical Facilities Utilization Reporting System improves upon a manual reporting system that the state local health planning councils had been administering for over 25 years. This system consists of two databases, the Hospital Utilization and the Nursing Homes. The Hospital Utilization database collects detailed inpatient and emergency department data from hospitals across the state. The Nursing Home Utilization database tracks admissions and patient days by payer source. These data sets are accessible online, thus improving program efficiency and overall functionality including utilizing data to make capacity and quality related decisions. The database has the ability to generate 39 exportable and/or ready-to-print reports. It was expanded to become a strategic planning tool for health care administrators to assess variances in utilization. Hospital and Nursing Home Utilization reporting is required by state statute and is delivered to the Agency for Health Care Administration on a quarterly basis.

The PQI provides county-level data that identifies hospitalizations and emergency department visits that may have been preventable with the utilization of high quality primary and preventive care. Pediatric Quality Indicators/Avoidable Admissions (PDI) provides county level data that identifies pediatric hospitalizations and emergency department visits that may have been preventable with the utilization of high quality primary and preventive care.

The DRG Data Warehouse is a decision support tool for health care providers and planners. It allows the user to quickly run customized reports by hospital medical services such as cardiology or orthopedics including DRG level detail by selected hospitals in an area using the Florida Agency for Health Care Administration (AHCA) hospital inpatient database.

These databases provide health care practitioners, planners, researchers, and policy-makers across the state with valuable community-planning resources to target initiatives, set benchmarks to increase health care access and quality, and identify target areas for quality improve [1].

The overall aim of this project was to evaluate CBI's capability to bridge the gap between BRHPC's data sources and the various end-users who need the data for analysis and, ultimately, for making informed decisions.

\section{Materials and Methods}

$\mathrm{CBI}$ is a powerful set of tools that has the potential to assist in the planning and coordination of the health care services. The project was designed to reflect the System Development Life Cycle (SDLC) development methodology, which consists of five stages: planning, analysis, detailed system design, implementation, and support. The SDLC describes activities and functions that all systems developers perform, regardless of which approach they use [2]. 
As part of the planning stage of the SDLC development methodology, BRHPC permitted the assessment of the existing informatics set-up of the FHDW, so that a thorough gap analysis could be performed. The analysis yielded major drawbacks in the initial set-up of BRHPC's informatics solution. The database infrastructure (systems and connectivity) prevented the high utilization of the large amount of health care data available for analysis and use. Figure 1 illustrates the gap in data utilization between the FHDW and the state's public health service decision makers, health care planners, hospitals, and the public at large.

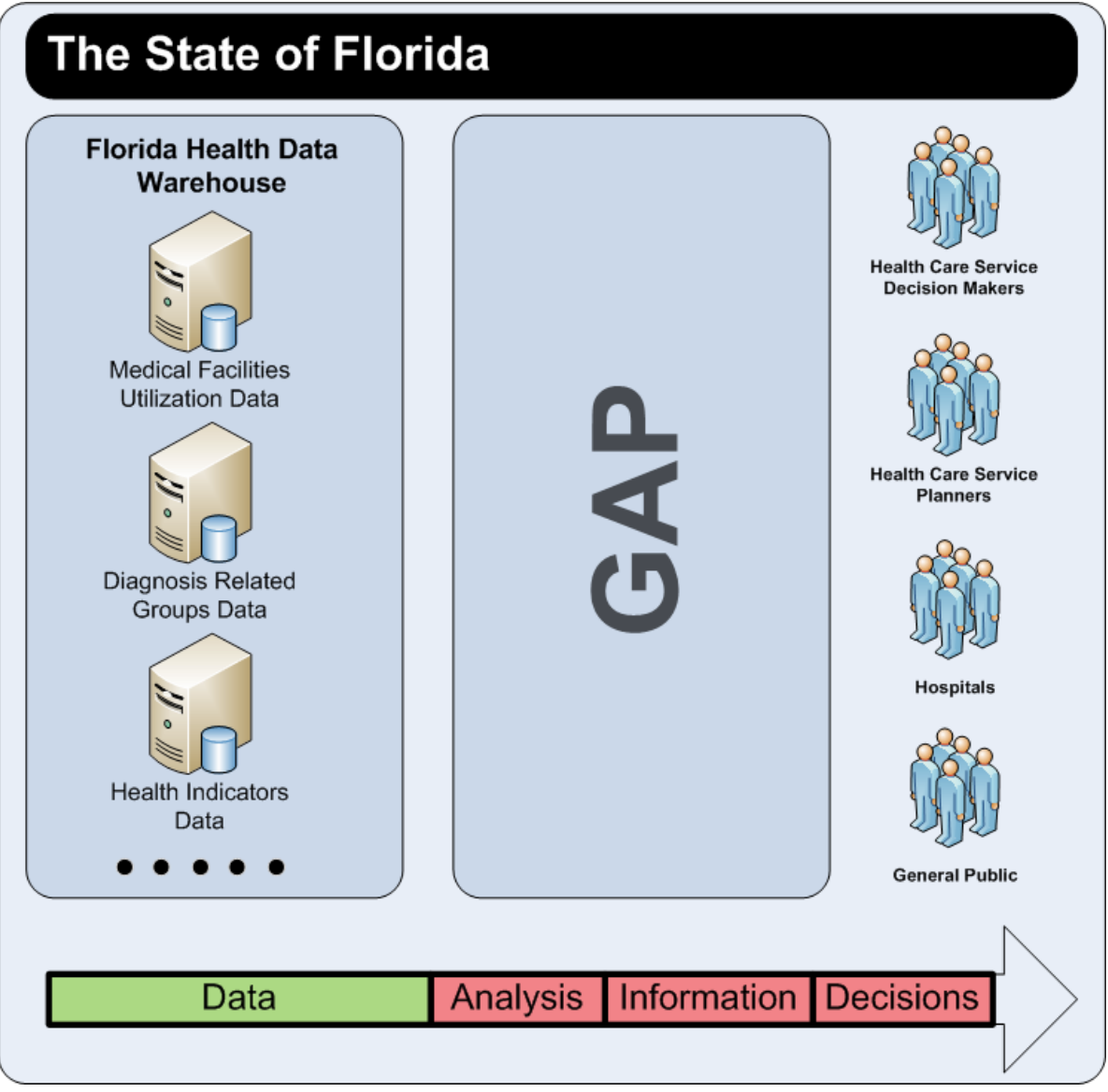

Figure 1: Health care data utilization gap between the FHDW and relevant stakeholders

The state's decisions makers, health care planners, hospitals, and other groups have limited access to the data because of technical and practical barriers. These groups require the data to be analyzed and converted to information in order to be suitable for the decision making process. Conducting the data analysis offline whenever a decision is needed, or as part of normal supervision, requires technical resources that may not be available for many user groups. In addition, the offline analysis is inefficient because it is typically lengthy, time consuming, and has to be repeated every time the data source is updated. This method also adds a technical layer between the data and the user groups, which in turn increases the complexity of the data utilization process.

CBI systems have the ability to bridge the gap between the data and the users. CBI may be defined as a set of mathematical models and analysis methodologies that exploit the available 
data to generate information and knowledge useful for complex decisions-making process [3].The main components of any typical CBI system include Extraction, Transformation and Loading (ETL); the data warehouse that consists of Unified Dimensional Models (UDMs) and multidimensional data marts; and the analytical tools. This set of components was used in this project to overcome the said issues.

The ETL component was used to retrieve the data from the existing heterogeneous data sources, transform the structure of the data to suit data analysis and data mining, and archive the data in the data warehouse in a multidimensional format. The execution of this process was configured in a manner to ensure the tight synchronization between the data warehouse and the data sources.

The data warehouse was configured to host four developed UDMs: 1) the hospital utilization, 2) the nursing homes, 3) the DRG, and 4) the health indicators. Each UDM was developed with sets of dimensions and measures to reflect its source. The data warehouse also included the data in the sources, but in multidimensional format. This format is very powerful for data analysis, and it is widely used for analyzing large amount of data [4].

The analytical tools used in this project provided access to the information and knowledge generated by the CBI system as a whole. These tools are generally what end-users interact with as part of the CBI system. The dashboard tool is one of the major tools of CBI systems. It consists of screens that show sets of data analysis widgets. Figure 2 show a dashboard of FHDWCBI system.

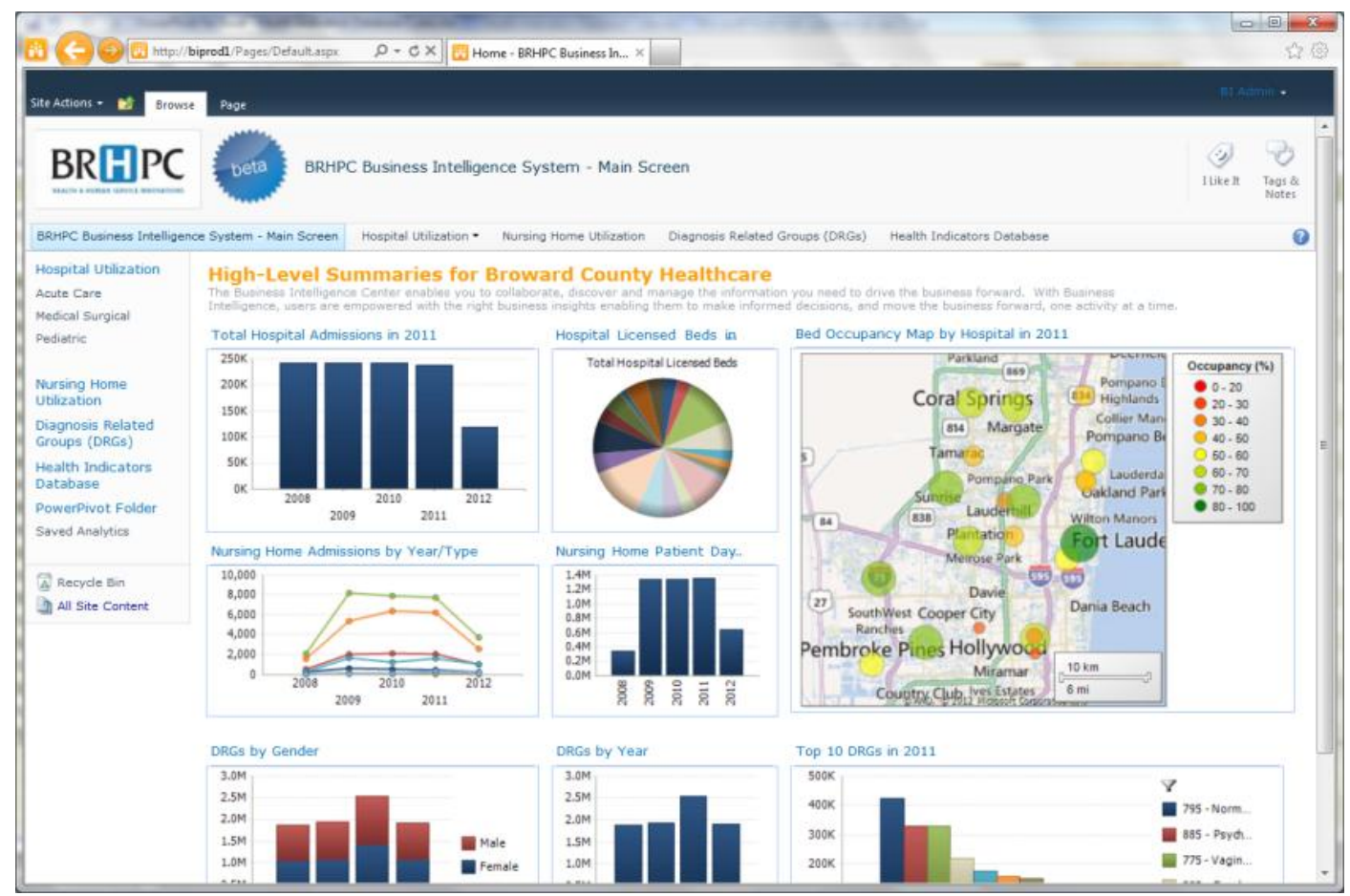

Figure 2: High-level summary dashboard of the BRHPC CBI System

There are also other tools that have been implemented in the FHDW's CBI system, including analytical reports and UDM access utilities. These tools allow end-users access to the UDMs and the multidimensional database for easy data analysis, without the need for technical skills. The 
data analysis can be done interactively and can be saved on the system for future retrieval. This is a major advantage as it gives end-users the ability to analyze data directly.

Upon the approval of the planning stage proposal, a project plan covering all the other stages of the SDLC (analysis, detailed system design, implementation, and support) was developed. The complete project plan consisted of three main parts: 1) project preparation; 2) system development and implementation; and 3) project finalization. In order to simplify the project management and enable task dependences, the three parts were divided into ten phases.

The initial phase of the project plan started with the analysis stage of the SDLC methodology. The purpose of the system analysis phase is to build a logical model of the new system [2]. Meetings were held with different end-users in order to collect the site's requirements for the CBI system. In addition, various analytical reports, statistics were studied, access to the existing databases was obtained and analysis conducted in order to compile a clear understanding about the site's needs. After the data collection process was completed, an analysis was done using the collected data and the results were incorporated into a demo system. The demo system had most of the capabilities that were needed to fulfill the end-user requirements, and the system was built based on more than 30 percent of the data that existed at the FHDW. The analysis phase also involved the allocation of all of the needed resources for the CBI system development and implementation, including the acquisition of the back-end and front-end hardware and software. Moreover, the phase involved finalizing the site agreement that was necessary for formalizing the project between Nova Southeastern University (NSU) and BRHPC.

Once the analysis stage was completed, the system design, development, and implementation stages were initiated. The three stages were implemented for each phase of the project. Phase 1, the infrastructure implementation, involves the installation and configuration of the CBI system platform. This includes the back-end Operating Systems (OSs), the databases, and the CBI system components, which are the ETL, UDM service, analytical reporting service, and the CBI portal. Phases 2, 3, 4, and 5 are related to the design, development, and implementation of the CBI system components. After Phase 5, which is a comprehensive system testing and enhancement process, Phase 6was initiated. It consisted of four rounds, each starting with gathering unresolved issues and/or discrepancies as well as enhancing requests from a site's super-user, and implementing them accordingly. This step was critically important because it tremendously improved the system's functionality by removing issues before releasing it to the production environment for end-user use. After the four rounds of system testing and enhancement phase were completed, the system was released into the production environment.

In accordance with the fifth and final stage in the SDLC development methodology, a support plan was created to maintain the uptime and performance of the system. This was accomplished by compiling a complete system documentation - the Technical System Architecture (TSA). The TSA document describes the various components of the new system and the technologies used in the development and implementation [5]. It includes technical diagrams that describe the system design, as well as detailed information about the system servers, applications, services, databases, and user accounts.

It is anticipated that the project will continue to grow in the years to come. As such, the system will eventually need to be scaled out in order to maintain the targeted system performance. The expansion plan will cover the system's two main components, the Microsoft SQL Server and the Microsoft SharePoint. In addition, the BRHPC's Information Technology (IT) team has been 
prepared to handle the system maintenance and administration activities. A technical training was delivered to the team. The training covered the TSA document and detailed steps for monitoring and maintaining system operation, user accounts, and backups.

\section{Discussion}

Vast amount of data is collected and stored by various health care organizations (HCO) throughout the country. This data is often underutilized as HCOs lack the clinical analytics tools necessary to turn the raw data into meaningful information in real time. CBI systems have the potential to offers $\mathrm{HCO}$ with valuable insights for improving patient care, tracking trends for medical research, and better controlling costs. In order for this to happen, the components of the CBI system must be designed properly.

The portal component of the CBI system BRHPC is based on Microsoft SharePoint 2010. The application is web-based, which allows end-users to access it from any computer connected to the Internet using standard web browsing applications.

Once valid credentials are provided, the portal will display the main screen. Regardless of the user access level, there are three main zones in all screens of the portal: the top control and navigation zone, the side navigation zone, and the analytics zone, as shown in Figure 3.

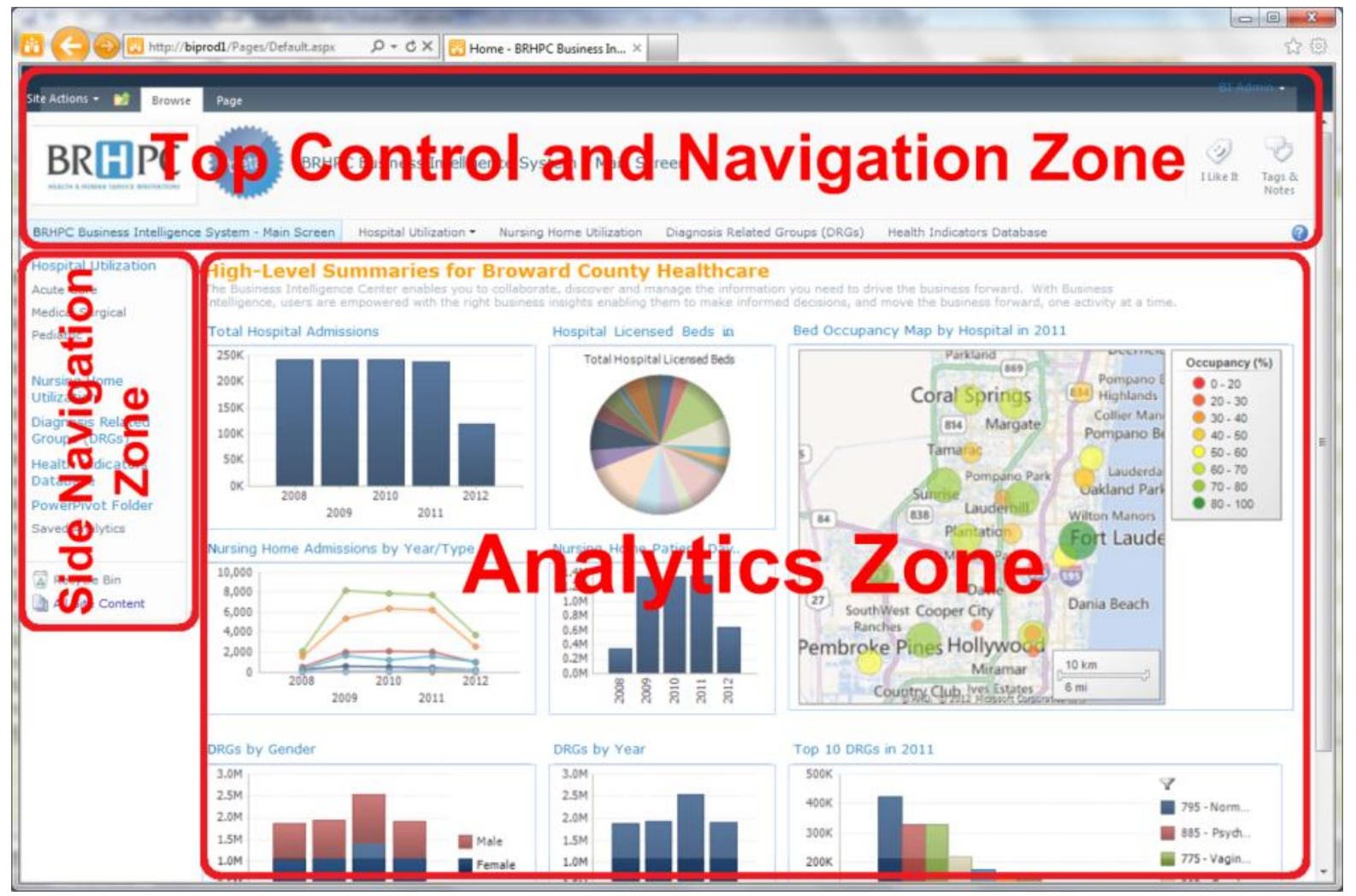

Figure 3: Main screen of the BRHPC CBI Portal

The top control and navigation zone includes a menu for navigation through the different dashboards within the system. It also includes links for controlling tags and notes about the dashboards, in addition to allowing the end-user to logout from the system. The side navigation 
zone also has a menu for navigation through the different dashboards. Additionally, it has a Recycle Bin link that allows restoring of deleted custom analytics by the user. The analytics zone encapsulates dashboards that display the analytical graphs, analytical maps, scorecards, and analytical reports. In some dashboards, there are tools that allow the end-user to access the UDMs, analyze, and save the result within the system or on the end-user's computer.

The main screen of the CBI portal displays a high-level summary of all analytics in the system. Seven analytical graphs and one analytical map are part of the main screen of the portal. The analytical graphs are interactive and allow the end-user to analyze the information beyond the layout that was developed by default. For example, if an end-user is interested in viewing the details of a year in the Total Hospital Admissions graph, he or she only need to click on the year and the portal will show the admission data by month. The graph can also be enlarged to a full screen size when the title is clicked. To analyze the data of the graph by the available dimensions, the end-user can right-click on the data bar of interest and then select the Decomposition Tree tool. This tool enables the end-user to drill down through the data easily and interactively, as shown in Figure 4.

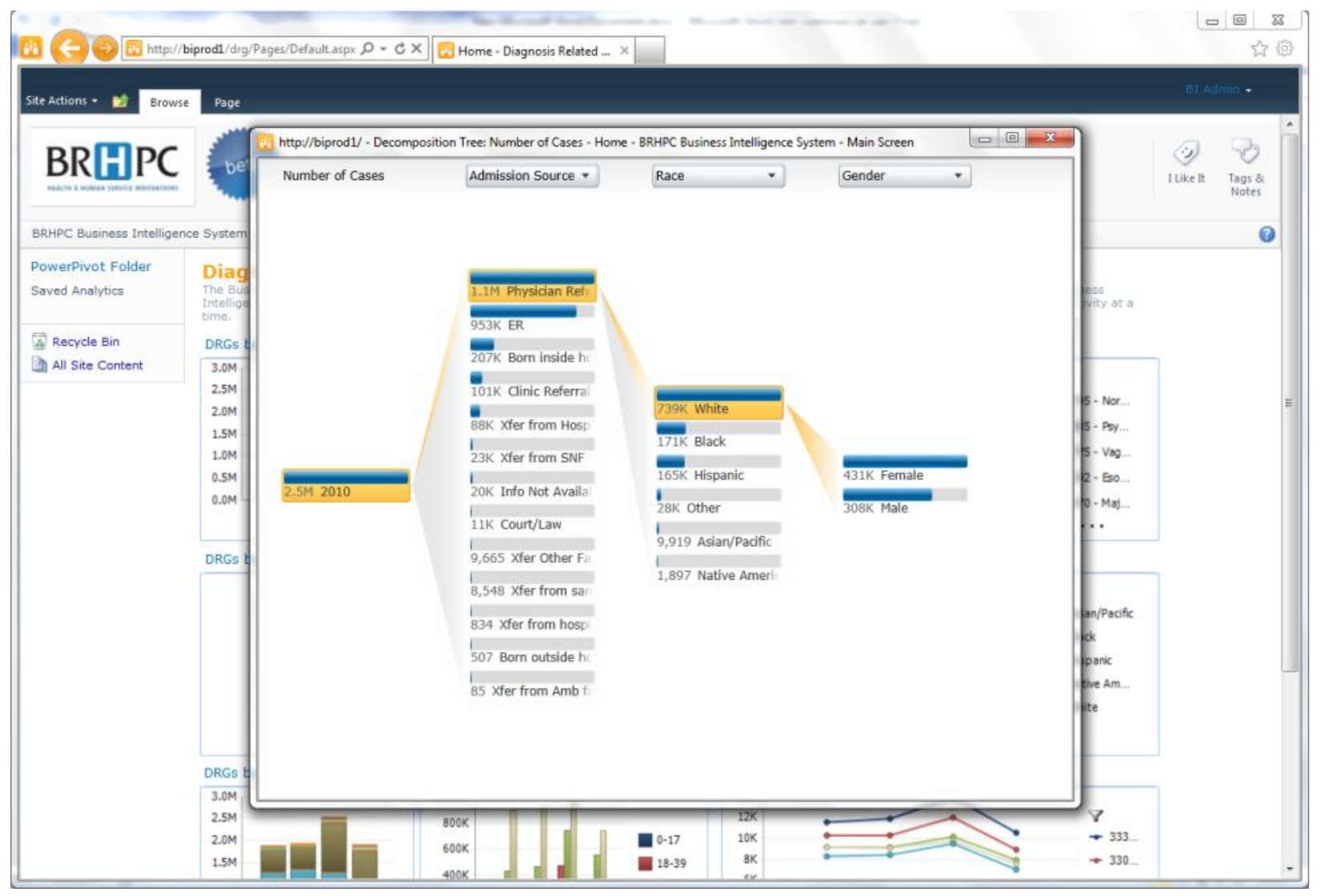

Figure 4: Drilling-down through the health care data

The tool sorts the dimension attributes based on their measure values. Graphical and numerical indicators are also some of the useful tool features, as they indicate the share of each dimension in comparison to the overall measure. A number of the web pages in the CBI portal have a section at the bottom called Data Cubes. This section has a number of tools that can be used to access the UDMs in the CBI system. The first option is PowerPivot, which is a tool that allows Online Journal of Public Health Informatics * ISSN 1947-2579 * http://ojphi.org * 6(2):e182, 2014 
the end-user to access UDMs and analyze them in an easy manner. The look and feel of this tool is similar to the popular office software, Microsoft Excel, which shortens the learning curve for the new end-users. Nonetheless, if the layout of the model needs to be modified, the tool allows end-users that have the necessary permissions to open the model in Microsoft Excel, modify it, and save it to their personal folder.

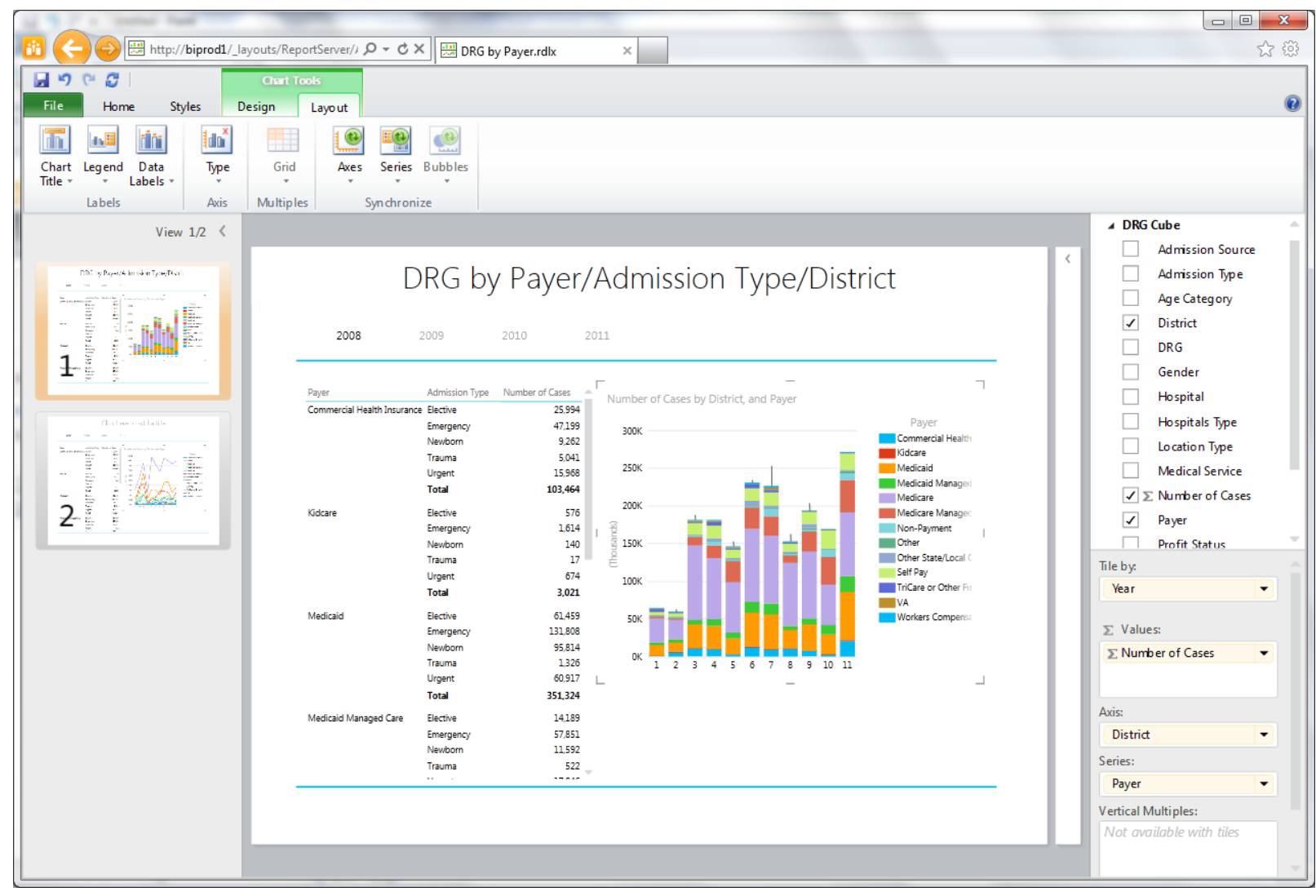

Figure 5: Sample analytical graph

On some modules of the CBI, such as the Hospital Utilization, there is an additional section at the bottom of the screen called Analytical Reports. A number of pre-designed reports can be found in this section. When one of the analytical reports is opened, default parameters are used to run the report. However, the reports allow modifying of certain parameters and re-running the report based on the new parameter configuration. One of the useful features is the ability to print the report or export it in different common formats.

Analytical reports also have a function called data alerts, which is a data driven notification solution that helps the end-user to be informed about the report's data that is of interest or importance at any given relevant moment. By using data alerts, the end-user no longer has to seek out information as it gets automatically delivered based on user specifications. Data alert messages can be sent by email or through short message service (SMS), i.e. text messages. Depending on the importance of the information, the end-user can choose to send messages more or less frequently, and only when results change. The end-user has the option to specify multiple email recipients to keep others informed or to enhance efficiency and collaboration among 
various stakeholders and participants. The configuration screen of data alerts is shown in Figure 6.

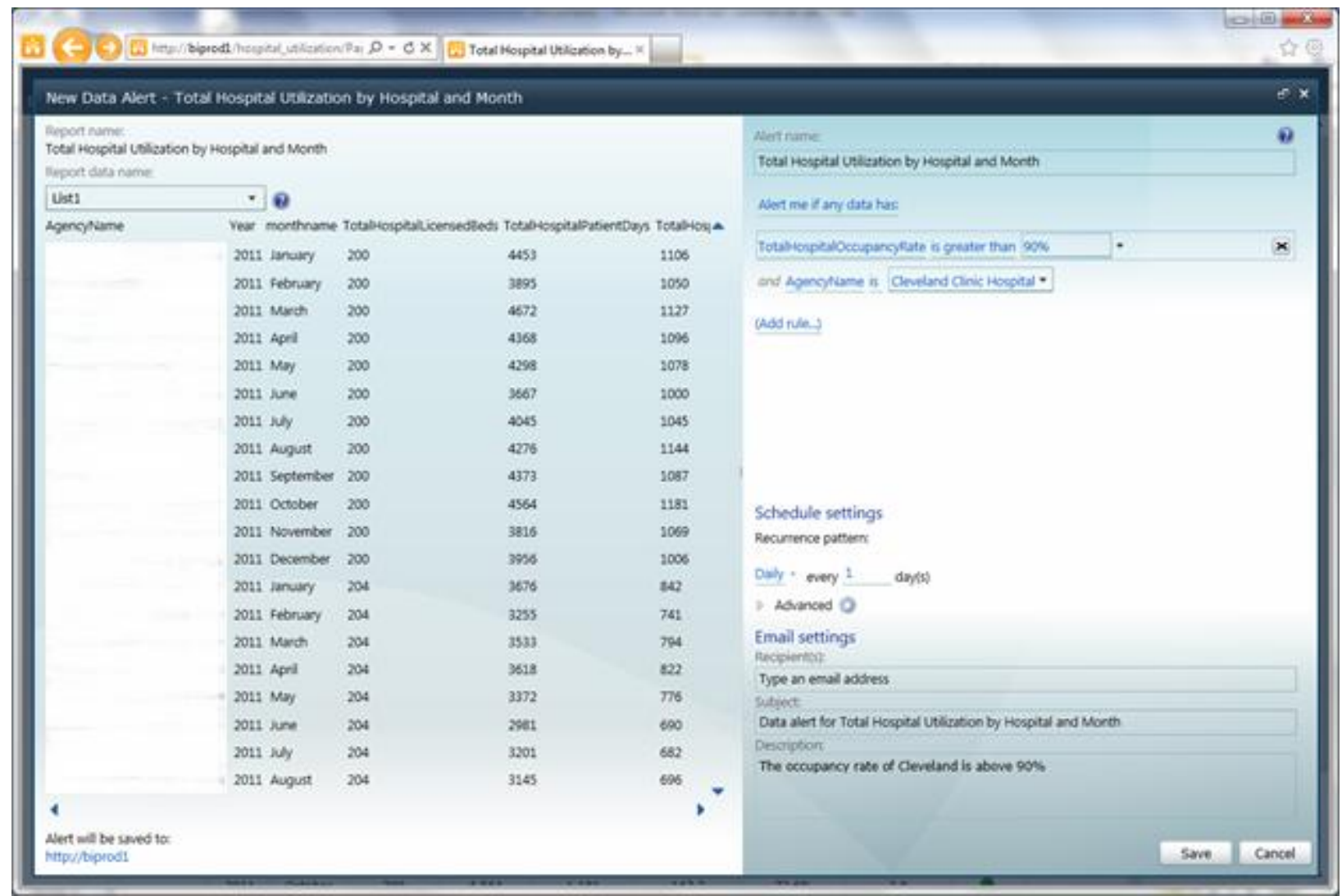

Figure 6: Configuration screen of data alerts

As a data alert owner, the end-user can view information, delete, and edit data alert definitions. An alert has only one owner, the end-user who created it. CBI system administrators can manage data alerts at the site level. They can view lists of alerts by each site user and delete alerts as needed.

Report subscription is another function in analytical reports that allow reports to be emailed to end-users based on schedule. This function is different because it is triggered only by time, not by changes in the data. With report subscription, the end-user can configure the report to be emailed to one or more email addresses. The report can be emailed in different formats, including comma-delimited (CSV), PDF, and TIFF image.

\section{Results}

Although the CBI system has just been released to the FHDW environment, it has already yielded a number of outcomes. The system provides much of the information needed to develop county health plans, which typically consists of hundreds of pages and requires months to compile. Unlike the county health plan, the CBI data is up-to-date and can be compiled and formatted in minutes. In addition, end-users can configure certain reports to be emailed to them based on a schedule or based on certain changes in the data. 
The system also has shortened the time needed to analyze the data, or transform it to information, and prepare it for decision making processes. Hospital planners are now able to get the data transformed to information on demand whenever they display one of the CBI portal dashboards. They even have the ability to modify/adjust the information to further fit the situation on hand. Typically, a number of data analysis professionals were needed to perform such processes and make decisions. The CBI system has helped eliminate this layer, which was not only costly but also time consuming.

In addition to the immediate outcomes just described, there are a number of anticipated advantages to BRHPC specifically, and the state of Florida generally. The advanced data analysis capabilities of the system are expected to improve the coordination and distribution of health care resources across the state. The quality of health service is also expected to be enhanced, as the system provides the ability to automate tracking issues in the delivery of health care services and reporting them to the relevant personnel. The system is also expected to enhance the health care planning for hospitals, health planning agencies and the state. The data mining component of the system has a number of prediction models that can assist in the planning process.

\section{Conclusion}

Vast amount of health care data is being collected and maintained nationwide, statewide, and within counties in the United States. However, there is a typical technological gap that exists between the data and users who need access to the data in order plan and coordinate health care services in the area. In the state of Florida, CBI has been developed and implemented in order to bridge the divide, and soon after, the solution yielded a number of positive outcomes. Based on these results, we suggest CBI as a solution for similar situations in other set-ups.

\section{Limitations of the Study}

It is important to highlight that the study has not been extended to evaluate the end-users' experience and the skill levels with the implemented solution. Without the end-users' acceptance and familiarity with the system use, the value of the solution can be significantly compromised [6]. Nonetheless, qualitative research methods such as focus groups, interviews and surveys can be used to collect information about the end-users' impressions toward the solution as well as their levels of ability to use the system. The outcomes of such research can determine the overall impact of the system and also assist in customizing it to meet the end-users' needs.

Another limitation was related to the changes of the coding system requirements. The CBI system was built based on standard coding systems such as DRG, the International Classification of Diseases $9^{\text {th }}$ Revision (ICD-9) and the Current Procedural Terminology (CPT). However, starting from October 1, 2014, healthcare providers will be required by the Centers of Medicare and Medicaid (CMS) to submit their claims using ICD-10 [7], which is the newer revision of the coding system that is used by the CBI. This study did not cover how the CBI system will handle the difference in coding between the archived data and the new data that fulfill the new coding requirements. 


\section{Acknowledgements}

The product of this research work would have not been possible without the help and support from the Broward Regional Health Planning Council and Nova Southeastern University. The authors are grateful for the opportunity provided and assistance received to help make this project a reality.

\section{References}

1. Broward Regional Health Planning Council. About BRHPC. n.d. [cited Nov 2012]; Available from: http://www.brhpc.org

2. Shelly GB, Rosenblatt HJ. Systems Analysis and Design. 2012, Boston, MA: Course Technology.

3. Vercellis C. Business Intelligence: Data Mining and Optimization for Decision Making. 2011, West Sussex, UK: John Wiley \& Sons Ltd.

4. Jensen C, Pedersen T, Thomsen C. Multidimensional Databases and Data Warehousing. 2010, San Rafael, CA: Morgan \& Claypool.

5. CCTA. Database and Physical Process Design. 2000, Norwich, UK: Crown.

6. Geddes DR. Quality Management Intensity during IS Development: Does it Influence EndUser Satisfaction? 2007, Ann Arber, MI: ProQuest Information and Learning Company.

7. Centers for Medicare and Medicaid. FAQs: ICD-10 Transition Basics. 2013 [cited Mar 2014]; Available http://www.cms.gov/Medicare/Coding/ICD10/Downloads/ICD10FAQs2013.pdf 